\title{
A Difraction Element Used to Evaluate the Depth of Bedding of Nano-Sized Radiating Objects
}

\author{
V.G. Volostnikov ${ }^{1,2}$, E.N. Vorontsov ${ }^{1}$, S.P. Kotova ${ }^{1,2 a}$, N.N. Losevskiy ${ }^{1,2}$, and D.V. Prokopova ${ }^{1,3}$ \\ ${ }^{1}$ Lebedev Physical Institute RAS, 221, Novo-Sadovaya Str., Samara 443011, Russia \\ ${ }^{2}$ S.P. Korolyov Samara State Aerospace University 34 Moskovskoye Shosse, Samara 443086, Russia \\ ${ }^{3}$ Samara State University, 1 Academician Pavlov Str., Samara 443011, Russia
}

\begin{abstract}
The paper describes a method to calculate the diffraction optical element (DOE) capable of forming a two-lobe light field rotating under its propagation. The basis of the approach is an iteration procedure where the zero-order approximation is defined with the aid of the spiral beams optics. The experimental results are offered on the formation of light fields under consideration by means of a liquid-crystal spatial phase modulator of light, HOLOEYE HEO-1080P.
\end{abstract}

Keywords: rotating light fields, spiral beams.

The problem of evaluating the depth of bedding of nano-sized radiating objects is topical for the fluorescence microscopy. For these purposes the approach based on the use of special optical elements seems to be rather promising. Such elements transform the radiation of a point light source into a light field with the structure within the observation plane depending on the distance from the object. A particular attention is paid to the elements transforming the radiation of a point light source into a field with two clear-cut maxima of intensity (two-lobe field).

These maxima are diametrically opposite to each other and occupy a definite space in the observation plane, depending on the distance from the object. The analysis of the maxima positions makes it possible to definitely determine the coordinates of the object observed. Within the frames of this work a modification of the iteration method is considered aimed to calculate the diffraction optical element that is responsible for the transformation described above.

A distinctive feature of the offered method consists in the application of principles of the spiral beams optics that have been earlier studied in detail [1]. A theoretical outline of such fields allows an analytical search of beams with pre-determined intensity distributions and parameters of the rotation under propagation $[1,2]$.

It was sufficient for the given task to consider the simplest type of spiral light beams, i.e. the sum of Laguerre-Gauss modes $\left(L G_{n, m}\right)$. Under the present study a beam of the type:

$$
F=L G_{0,0}+L G_{1,2}+L G_{2,4}+L G_{3,6}+L G_{4,8}
$$

has been investigated. The selection of indexes $(n, m)$ resulted in the beam rotation by $2 \pi$ [2] at propagation.

\footnotetext{
${ }^{a}$ Corresponding author: kotova@fian.smr.ru
} 
The beam phase (1) was used as the zero-order approximation for the iteration procedure of the DOE calculation. The approach described in [3] was taken as the basis of the calculation algorithm. But in our case the intermediate expansions (decompositions) by Laguerre-Gauss modes were not carried out. As the result, the procedure was simplified and the calculation time was redused. These simplifications had no influence on the element difraction parameters.

The initial phase of the spiral light beam (1) and the phase of DOE calculated with the offered approach after 6 iterations are shown in Fig.1. The calculated values of the intensity distributions over different cross-sections along the propagation axis are given in Fig.2 (upper row). The difraction efficiency of the element defined as the ratio of energy located in maxima and total beam energy in the Fourier plane is $\sim 62 \%$. By increasing the iterations number up to 10 , it grows insignificantly ( $66 \%)$.

In experiments on the light fields formation a solid-state laser was used with the power up to 50 $\mathrm{mW}$, and $\lambda=532 \mathrm{~nm}$. An expanded and homogeneous by intensity, laser beam was directed onto a multi-pixel liquid-crystal spatial phase modulator of light, HOLOEYE HEO-1080P, where the signal was fed to, ensuring the formation of the calculated phase distribution. The intensity distribution of the obtained light field and its alteration along the Z-axis was registered with the horizontal microscope and digital camera.

The results of the experiment are presented in Fig. 2 (bottom row). The experimentally measured difraction efficiency of the beam defined as the ratio of the signal located in maxima and the total beam signal registered by means of the digital camera was $\sim 56 \%$.
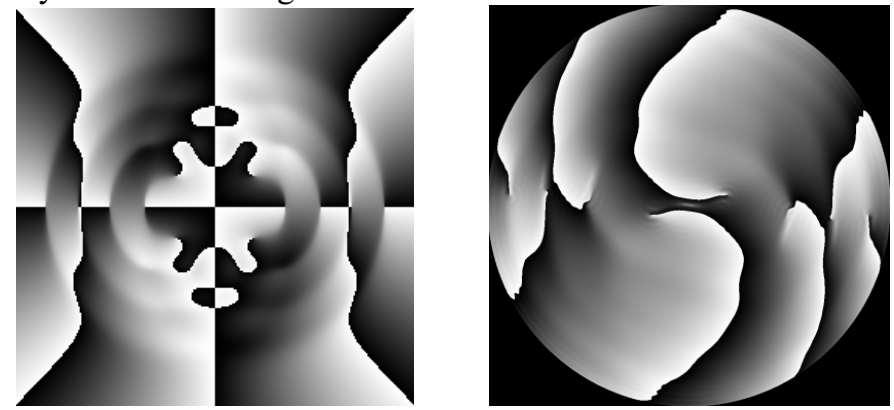

Figure 1. Phase of the spiral beam and DOE calculated on its base.

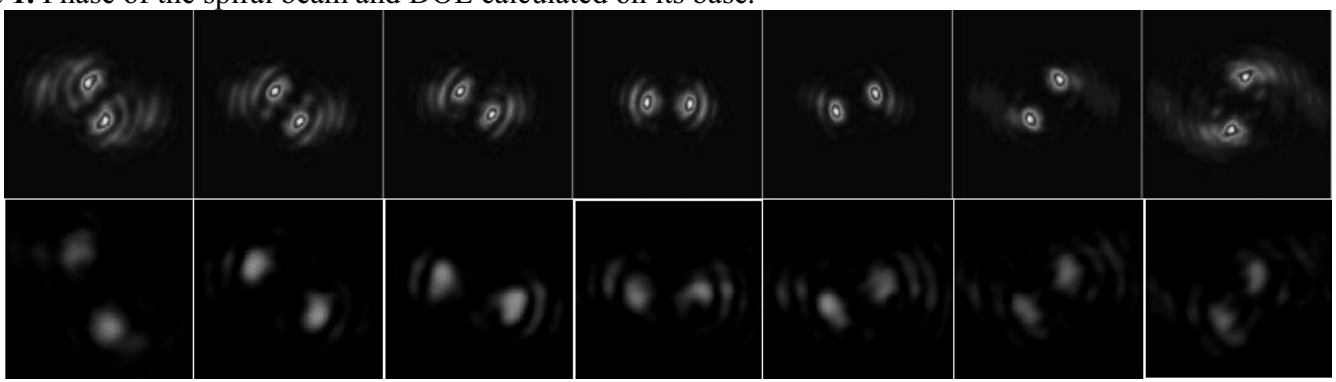

Figure 2. The intensity distributions of the formed fields in cross-sections perpendicular to Z-axis for the DOE calculated with the iteration method after 6 iterations. In the upper row - calculated intensity distributions, in the bottom row - those experimentally obtained.

The research project was supported by the Program for Basic Researchers of the Physical Sciences Department of RAS "Fundamentals and experimental realization of promising semiconductor lasers in the interests of industry and technology".

\section{References}

1. E.G.Abramochkin, V.G.Volostnikov, Uspekhi Fiz. Nauk 174. No. 12. P. 1273-1300 (2004)

2. E.G.Abramochkin, V.G.Volostnikov, Modern Optics of Gaussian beams. (M.:FIZMATLIT, 2010)

3. S.R.P. Pavani, R. Piestun, Optics Express. 16. N. 5. P. 3484-3489 (2008) 